\title{
ACTIVE ABSORPTION OF NONLINEAR IRREGULAR WAVES
}

\author{
Lykke Andersen, T. ${ }^{1}$, Clavero, M. ${ }^{2}$, Eldrup, M. R. ${ }^{1}$, Frigaard, P. ${ }^{1}$, Losada, M. ${ }^{2}$
}

\begin{abstract}
In the present paper, the performance of active absorption systems based on nearfield surface elevation measurements is studied. Based on linear wavemaker theory the performance of such systems can easily be calculated for linear waves. A recent study demonstrated that bound superharmonics in regular waves is also well absorbed by such system and has a re-reflection similar to a linear component. However, the performance of active absorption systems on nonlinear irregular waves has never been studied. In the present paper the absorption of bound sub and superharmonics in bichromatic and irregular waves is examined based on new model tests. The conclusion is that also in irregular waves the bound harmonics are well absorbed by the studied active absorption system.
\end{abstract}

Keywords: active absorption; physical model tests; nonlinear waves; reflection; wave analysis

\section{INTRODUCTION}

Accurate physical modelling requires in most cases to deal with the wave reflections from the structures in the model testing facility in a proper way. Reflected waves from structures in wave flumes and basins will without active absorption become re-reflected at the wavemaker and thus contaminate the desired incident waves. This problem is especially visible when testing highly reflecting structures like caisson breakwaters, where the re-reflections will cause energy build-up at the resonance frequencies. However, also when testing less reflecting structures the contamination will occur but to less extend. When testing mildly reflecting structures the most typical, and also most critical contamination, is the built up of long wave energy (seiches). The result is too much long period energy and unrealistic response of structures sensitive to long waves, for example overtopping on rubble mound breakwaters. Thus, it is important that the active absorption system is also effective for long waves. Therefore, effective active absorption is needed to increase the reliability of physical model tests by controlling spurious oscillations at different frequencies.

In long flumes, re-reflections at the wavemaker can be avoided by terminating the test before rereflections occur as done in many studies before active absorption systems became common. However, in short flumes or in irregular sea states this option is not possible or at least not practical.

Active absorption systems are based on linear wavemaker theory and their performance on nonlinear waves has not been studied until recently. The present paper extends the recent studies for nonlinear regular waves by Lykke Andersen et al. (2016) to nonlinear bichromatic and irregular waves.

\section{APPLIED ACTIVE ABSORPTION SYSTEM}

Many types of active absorption systems have been proposed throughout the years. Most systems are based on measurements in the nearfield, but an alternative method based on measurement of surface elevations or velocities in the farfield was established by Frigaard and Christensen (1994), Christensen (1995) and Hald and Frigaard (1996, 1997a, 1997b). These farfield based systems give more time to react and are thus simple to implement in causal filters and lead to systems that are quite robust to errors. However, the farfield systems are difficult to extend to short-crested wave conditions and also have significant problems with absorption of the bound superharmonics in nonlinear deep water waves.

For these reasons most laboratories still prefer systems based on measurement in the nearfield. The most typical nearfield feedback signal for the active absorption loop is the surface elevation on the paddle face. This was first demonstrated by Milgram (1965, 1970) who designed an active wave absorber. Later similar systems were designed and used in many laboratories, cf. Gilbert (1978), Kostese (1984), Schäffer et al. (1994), Klopman et al. (1996), Schäffer and Jakobsen (2003) and Lykke Andersen et al. (2016).

Alternative systems with measurement of forces on the wave boards were presented by Salter (1981), Chatry et al. (1998) and Spinneken and Swan (2009). These methods have though so far not achieved as wide a frequency band with low re-reflection coefficient as the systems based on surface elevation measurements.

The method chosen by most laboratories is based on measurement of surface elevation in the nearfield and such system is also examined in the present paper. The tested system is the one presented

\footnotetext{
${ }^{1}$ Dept. of Civil Eng., Aalborg University, Denmark, Thomas Manns Vej 23, DK-9220 Aalborg Ø, Denmark

${ }^{2}$ Andalusian Inst. for Earth System Research, Universidad de Granada, Avd. del Mediterráneo, 18006 Granada, Spain
} 
in Lykke Andersen et al. (2016) which is implemented in the AwaSys software, cf. Aalborg University (2018a). The system is based on fully linear wavemaker theory (including evanescent modes) and leads to the following target frequency response function:

$$
H(f)=\frac{1}{i C_{0,2 D}\left(\exp \left(i k d_{W G}\right)-2 \cos \left(k d_{W G}\right)\right)-D\left(d_{W G}\right)}
$$

where $C_{0,2 \mathrm{D}}$ and $D$ are the Biésel far- and nearfield transfer functions (see details in Lykke Andersen and Frigaard (2014)). $k$ is the wave number of the progressive wave, $d_{\mathrm{WG}}$ the gap between the wave board and the wave gauge.

Lykke Andersen et al. (2016) showed that a causal digital FIR filter can be designed, and they provided an iterative filter design procedure. This procedure involves performance optimization in a desired frequency band, i.e. minimizing the difference to the target transfer function given in Eq. 1. For frequencies outside that band, the important issue is that the system is stable.

Based on the actual frequency response function $\widetilde{H}(f)$ of the designed digital filter, the theoretical re-reflection coefficient of the wavemaker $\left(C_{\mathrm{r}}=H_{\mathrm{r}} / H_{\mathrm{i}}\right)$ can be calculated based on fully linear wavemaker theory, cf. Lykke Andersen et al. (2016):

$$
C_{r}(f)=\left|1-\frac{i 2 C_{0,2 D} \cos \left(k d_{W G}\right)}{1 / \widetilde{H}(f)-i C_{0,2 D} \exp \left(i k d_{W G}\right)+D\left(d_{W G}\right)}\right|
$$

The Lykke Andersen et al. (2016) system has many similarities with the system by Schäffer and Jakobsen (2003), but also a few important differences. Above transfer function is for example extended to cover cases where the wave gauge has a small gap $\left(d_{\mathrm{wg}}\right)$ to the paddle face, whereas Schäffer and Jakobsen (2003) assumed $d_{\mathrm{WG}}=0$. Moreover, Schäffer and Jakobsen (2003) used an IIR filter instead of a FIR filter, and they did not provide the filter design method. Both filter types lead to excellent active absorption performance, and thus the main advantage of the FIR filter is that any small error cannot accumulate in the filter. A consequence hereof is that the paddle drift of the system (response at zero frequency) will also always be finite with a FIR filter, which is usually not the case with IIR filters. For this reason, a zero forcing algorithm (filter) is needed in many systems built on IIR filters. This might be the reason for the observed better low frequency performance of the Lykke Andersen et al. (2016) system compared to the IIR based system of Schäffer (2001). This is important for the absorption of the long waves (for example reflected subharmonics) which is critical for the response of many structures.

Fig. 1 shows the theoretical performance curve based on Eq. 2 for a water depth of $0.7 \mathrm{~m}$. The figure demonstrates that it is possible to design a FIR filter that leads to low reflection coefficient in a wide frequency band $(0.15 \mathrm{~Hz} \leq f \leq 1.2 \mathrm{~Hz})$.

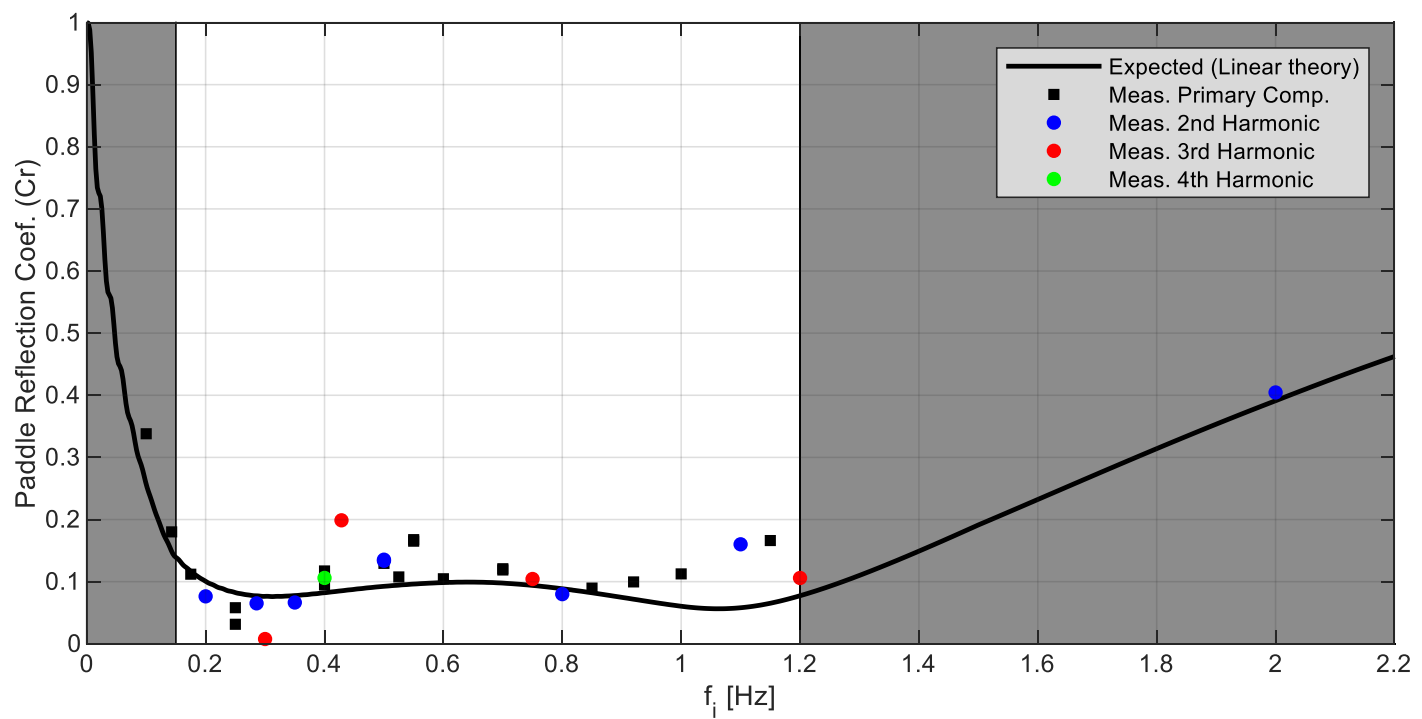

Figure 1. Performance of the Lykke Andersen et al. (2016) active absorption system in terms of re-reflection coefficient of the paddle. Theoretical curve based on linear wavemaker theory is compared to measurements with regular waves (based on data from Lykke Andersen et al. (2016)). 
Lykke Andersen et al. (2016) validated the theoretical performance curve for linear regular and irregular waves. Moreover, they studied the performance of the system on nonlinear regular waves, and they showed that the system was able to absorb superharmonics in highly nonlinear regular waves with similar performance as a linear component at the same frequency, cf. Fig. 1.

As demonstrated in Fig. 1 the Lykke Andersen et al. (2016) system has the correct frequency dependent transfer function in a quite wide frequency range, and thus the system is applicable also to irregular waves. However, the performance for nonlinear irregular waves has not been investigated previously and was thus the purpose of the present study. This includes accurate generation of nonlinear irregular waves and quantification of the absorption performance of the system for bound sub and super harmonics in irregular waves. For this purpose, additional tests have been performed using state-of-theart nonlinear wave generation and analysis methods.

\section{PHYSICAL MODEL TESTS}

In order to quantify the performance of the active absorption system model tests were carried out in Atmosphere-Ocean Interaction Flume in Granada, Spain. A detailed description of the facility can be found in Clavero et al. (2013). The flume has wavemakers in both ends and is thus the ideal facility for studying the performance of the active absorption system. The test setup is identical to the setup used in Lykke Andersen et al. (2016). However, in between the two test series the control parameters in the wavemaker servo controller was optimized, and that led also to a slight improvement of the theoretical active absorption performance curve compared to the one presented in Fig. 1.

\section{Test Setup and Procedure}

The flume and the installed array of wave gauges are shown in Fig. 2. Wavemaker 2 was not generating waves, but only absorbing the waves generated by Wavemaker 1 using the Lykke Andersen et al. (2016) method. By calculating the reflection coefficient for every component in the sea state, the absorption performance of Wavemaker 2 could be studied. Wavemaker 1 was generating the waves and simultaneously absorbing any waves re-reflected from Wavemaker 2 . All tests were performed with a constant water depth $(h)$ of $0.65 \mathrm{~m}$. The width of the flume is $1.0 \mathrm{~m}$.

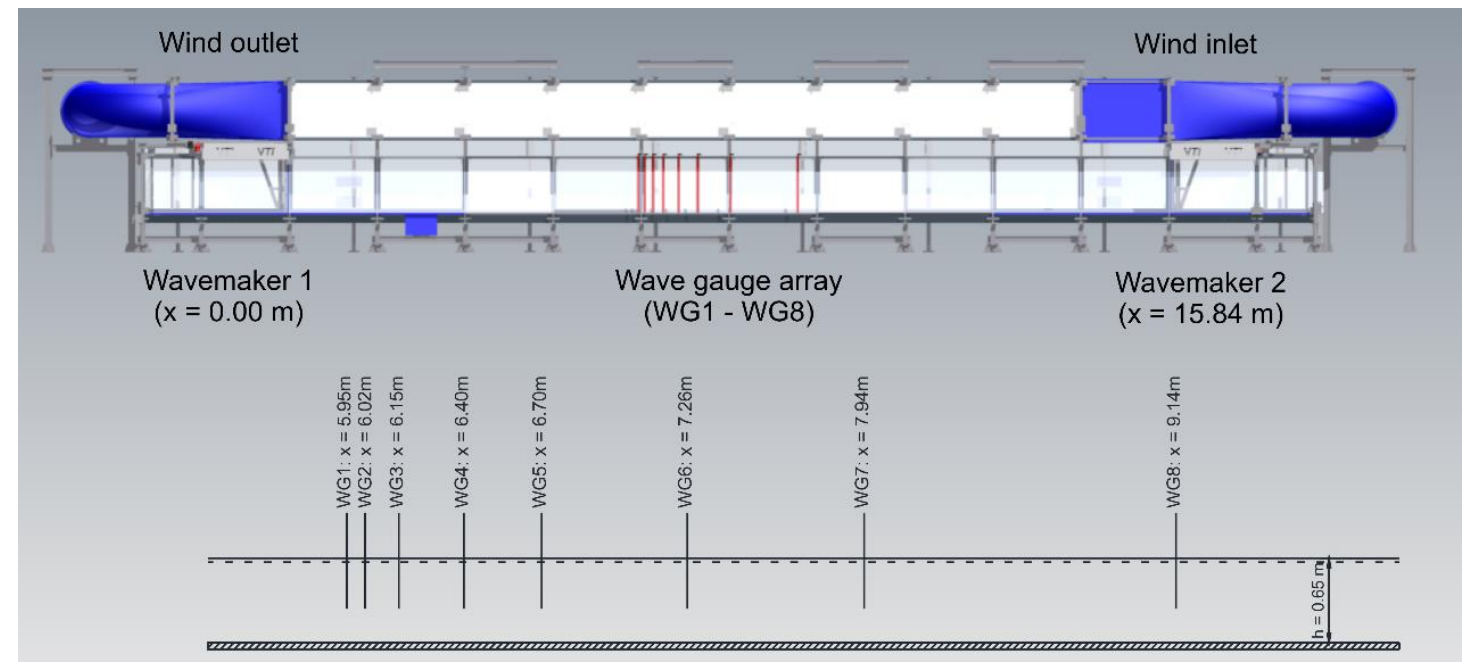

Figure 2. Model test setup including wave gauge positioning.

In order to minimize spurious free incident harmonic generation, it is necessary to correctly generate the bound harmonics. Therefore, the waves generated by Wavemaker 1 was synthesized using state-ofthe-art methods for nonlinear wave generation.

\section{Test Programme and Methodology for Bichromatic Waves}

Second order wavemaker theory (Schäffer and Steenberg, 2003) was used for all tests with bichromatic waves. For regular waves, Eldrup and Lykke Andersen (2018) suggested $S=1.5$ as the upper limit of the nonlinearity parameter for acceptable results with second order wavemaker theory. For irregular waves, they suggested an upper limit of $S=2.0$. For the bichromatic waves the nonlinearity parameter is in the present paper calculated with a frequency $f=\left(f_{\mathrm{n}}+f_{\mathrm{m}}\right) / 2$ and a maximum wave height $H=2\left(a_{\mathrm{n}}+a_{\mathrm{m}}\right)$ which leads to nonlinearity parameters in the interval $0.34 \leq S \leq 1.63$. Thus the most nonlinear case (BIC01) is on the limit of acceptable results with $2^{\text {nd }}$ order theory, but as it appears from Table 1 the second order wavemaker theory is valid for the remaining tests $(S \leq 0.9)$. 


\begin{tabular}{|l|c|c|c|c|c|}
\hline \multicolumn{6}{|l|}{ Table 1. Bichromatic wave test cases. } \\
\hline Test Name & $\begin{array}{c}f_{\mathrm{n}} \\
{[\mathrm{Hz}]}\end{array}$ & $\begin{array}{c}f_{\mathrm{m}} \\
{[\mathrm{Hz}]}\end{array}$ & $\begin{array}{c}a_{\mathrm{n}} \\
{[\mathrm{m}]}\end{array}$ & $\begin{array}{c}a_{\mathrm{m}} \\
{[\mathrm{m}]}\end{array}$ & $S$ \\
\hline BIC01 & 0.30 & 0.40 & 0.050 & 0.050 & 1.63 \\
BIC02 & 0.50 & 0.60 & 0.050 & 0.050 & 0.82 \\
BIC03 & 0.60 & 0.70 & 0.050 & 0.050 & 0.71 \\
BIC04 & 0.60 & 0.80 & 0.050 & 0.050 & 0.68 \\
BIC05 & 0.50 & 0.55 & 0.050 & 0.050 & 0.87 \\
BIC06 & 0.50 & 0.52 & 0.050 & 0.050 & 0.90 \\
\hline BIC07 & 0.30 & 0.40 & 0.025 & 0.025 & 0.81 \\
BIC08 & 0.50 & 0.60 & 0.025 & 0.025 & 0.41 \\
BIC09 & 0.60 & 0.70 & 0.025 & 0.025 & 0.35 \\
BIC10 & 0.60 & 0.80 & 0.025 & 0.025 & 0.34 \\
BIC11 & 0.50 & 0.55 & 0.025 & 0.025 & 0.44 \\
BIC12 & 0.50 & 0.52 & 0.025 & 0.025 & 0.45 \\
\hline
\end{tabular}

\section{Test Programme and Methodology for Irregular Waves}

In the present paper only the five most nonlinear irregular wave test will be reported. These tests have a nonlinearity parameter $1.3 \leq S \leq 3.3$ and thus second order wavemaker theory will for the main part of the tests lead to significant spurious free harmonics, cf. Eldrup and Lykke Andersen (2018) criteria. For this reason, the ad-hoc unified wave generation method by Zhang et al. (2007) was used for all cases. The MIKE21BW numerical model was used to generate the input for the ad-hoc unified generation method. The spectrum generated in the numerical model was JONSWAP with a peakenhancement factor of 3.3. This spectrum was in the numerical model generated at a depth of $1.4 \mathrm{~m}$ and shoaled up on a 1:100 foreshore to the depth of the physical model $(h=0.65 \mathrm{~m})$. Thus, the spectral shape generated in the physical model deviate somewhat from JONSWAP due to the shoaling process. The wave parameters generated in the physical model are shown in Table 2.

\begin{tabular}{|l|c|c|c|}
\hline \multicolumn{4}{|c|}{ Table 2. Irregular wave test cases. } \\
\hline Test Name & $\begin{array}{c}T_{\mathrm{p}} \\
{[\mathrm{s}]}\end{array}$ & $\begin{array}{c}H_{\mathrm{m0}} \\
{[\mathrm{m}]}\end{array}$ & $S$ \\
\hline SW01 & 4.0 & 0.075 & 2.25 \\
SW02 & 4.0 & 0.110 & 3.29 \\
SW03 & 3.0 & 0.075 & 1.33 \\
SW04 & 3.0 & 0.110 & 1.95 \\
SW05 & 3.0 & 0.150 & 2.65 \\
\hline
\end{tabular}

\section{Wave Analysis Methodology}

In order to characterize the performance of active absorption systems for nonlinear waves, it is necessary to be able to separate incident and reflected nonlinear waves. This involves also separation of the sub and superharmonics into incident and reflected parts. In order to separate the harmonics accurately, it is necessary to consider that bound and free components might be present and that they travel with different celerities. Moreover, amplitude dispersion is important when waves become nonlinear. In order to do this analysis accurately, it was thus necessary to develop new nonlinear methods that were presented recently by Lykke Andersen et al. (2017) for regular waves and extended to bichromatic waves by Lykke Andersen et al. (2018) and finally to irregular waves by Eldrup and Lykke Andersen (2019). In these papers, it is also demonstrated that predictions by linear reflection analysis methods might lead to very wrong results when applied to nonlinear waves. The new nonlinear methods have been implemented in the WaveLab software (Aalborg University (2018b)), and this software is used for all the present analyses.

The new nonlinear reflection separation methods will thus be used to quantify the reflection coefficient of wavemaker 2 which is absorbing the waves generated by wavemaker 1 . The absorption is based on the previously mentioned active absorption system, and thus its performance curve in terms of reflection coefficient as function of frequency can be validated for both linear and nonlinear waves. This analysis involves both the reflection of primary components and bound sub and superharmonics. 


\section{RESULTS FOR NONLINEAR BICHROMATIC WAVES}

The most nonlinear bichromatic test case is BIC01 for which the predicted incident and reflected waves are shown in Fig. 3. Time series are shown for the primary wave including all the bound harmonics as well as for the spurious free harmonics. The time series are shown with all components up to third order which is in agreement with the assumptions in the analysis procedure (Lykke Andersen et al., 2018). The figure demonstrates that the spurious free waves are small in comparison to the primary and bound waves.

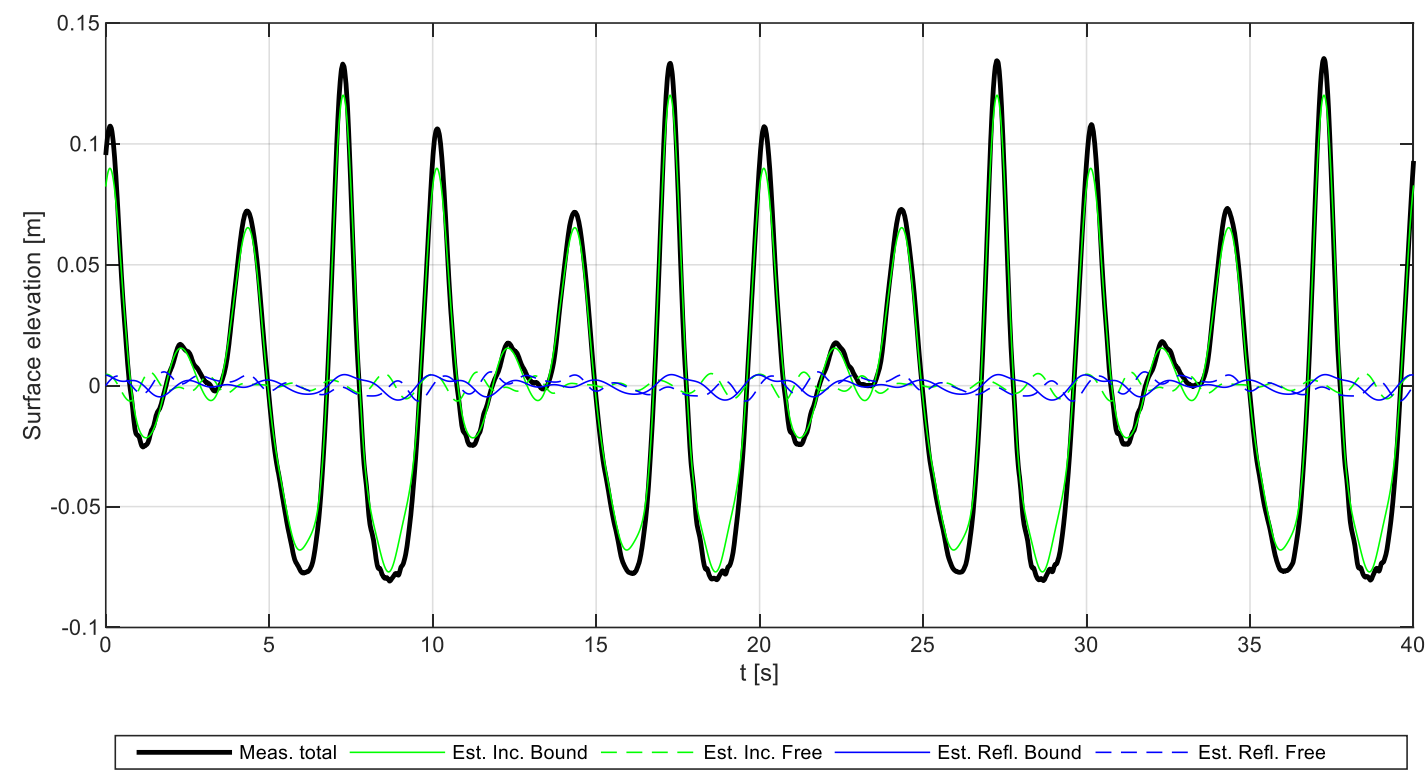

Figure 3. Measured total time series at WG1 $(x=5.95 \mathrm{~m})$ and estimated incident and reflected time series at the same position.

Additionally, Table 3 shows the amplitudes of the estimated components and also a comparison to the third order wave theory by Madsen and Fuhrman $(2006,2012)$. The table shows that at third order the free incident harmonics are not insignificant. For the second order components the second order wavemaker theory has effectively suppressed spurious free superharmonics as they are much smaller than the bound ones. A significant deviation from third order wave theory is only found for the third order bound sub harmonics which are highly overpredicted, due to singularities in the transfer functions by Madsen and Fuhrman $(2006,2012)$ as also found by Lykke Andersen et al. (2018). The estimated amplitudes of the other bound incident components are in fair agreement with third order wave theory.

The free and the bound components travel with different celerities and thus the total amplitude will vary throughout the flume. Therefore, the calculation of the reflection coefficient for each of the components is not straight forward. To include this modulation the reflection coefficient was calculated as the ratio of total reflected and incident amplitudes at the reflection line (at Wavemaker 2, $x=15.84$ $\mathrm{m})$. However, this means also spatial extrapolating of the results far from the measurement locations and thus small errors on the celerities of the bound and free components might cause significant uncertainty on the results. Therefore, it was chosen to calculate also the reflection coefficients based on total wave amplitudes in the middle of the wave gauge array (average of the wave gauge coordinates, $x=6.945 \mathrm{~m}$ ). Thus, in Table 3 two reflection coefficients appear for each component. Table 3 demonstrates that the reflection coefficient is low even for the higher harmonics. Moreover, for most of the components there is not significant difference between taking the reflection coefficient at the wave gauge array or at the wavemaker position. An exception is for $a_{\mathrm{n}+\mathrm{m}}$ for which an unphysical high bound reflected amplitude is predicted and thus the extrapolation outside of the wave gauge array leads to erroneous results. Another exception is for the third order component $a_{\mathrm{n}+2 \mathrm{~m}}$ at $1.1 \mathrm{~Hz}$ where the incident amplitude is less than $3 \mathrm{~mm}$ and some cross-modes were observed in the flume. The two lowest cross-mode frequencies are $0.87 \mathrm{~Hz}$ and $1.25 \mathrm{~Hz}$ for free waves of small amplitude. Actually, the third order bound component at $f=1.1 \mathrm{~Hz}$ has a wave length $L=2.15 \mathrm{~m}$ and is thus very close to the first cross mode $(L=2.0 \mathrm{~m})$. For this reason, results for frequencies above $0.85 \mathrm{~Hz}$ should be used with some care. For components with very small incident amplitude small errors like cross-modes might thus lead to large uncertainty on the calculated reflection coefficient. 


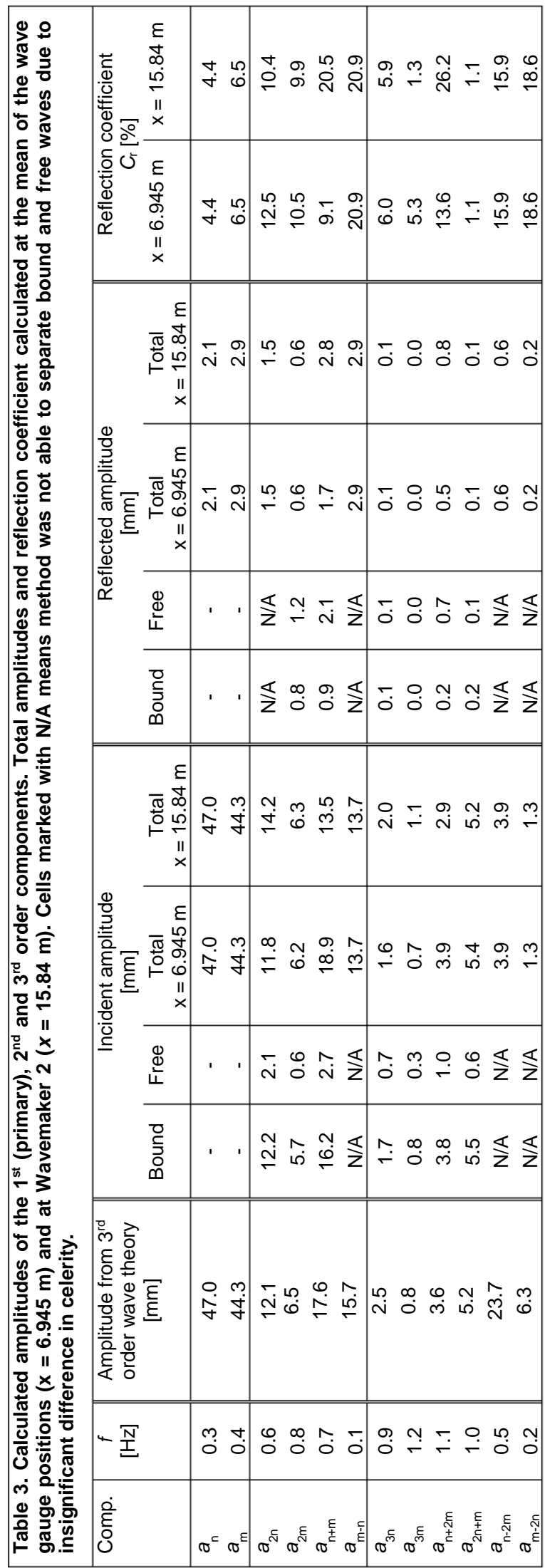


The reflection coefficients for the two positions is also plotted in Fig. 4 against frequency and compared with the active absorption performance curve based on linear wavemaker theory (Eq. 2). For most of the components the reflection coefficient is in very well agreement with the theoretical linear performance curve at both positions. The bound harmonics in the operational band $(0.15$ to $1.2 \mathrm{~Hz})$ has less than $20 \%$ re-reflection coefficient. Based on this test it seems fair to conclude that bound sub and superharmonics in bichromatic waves can be absorbed with almost similar performance as a free wave at the same frequency.
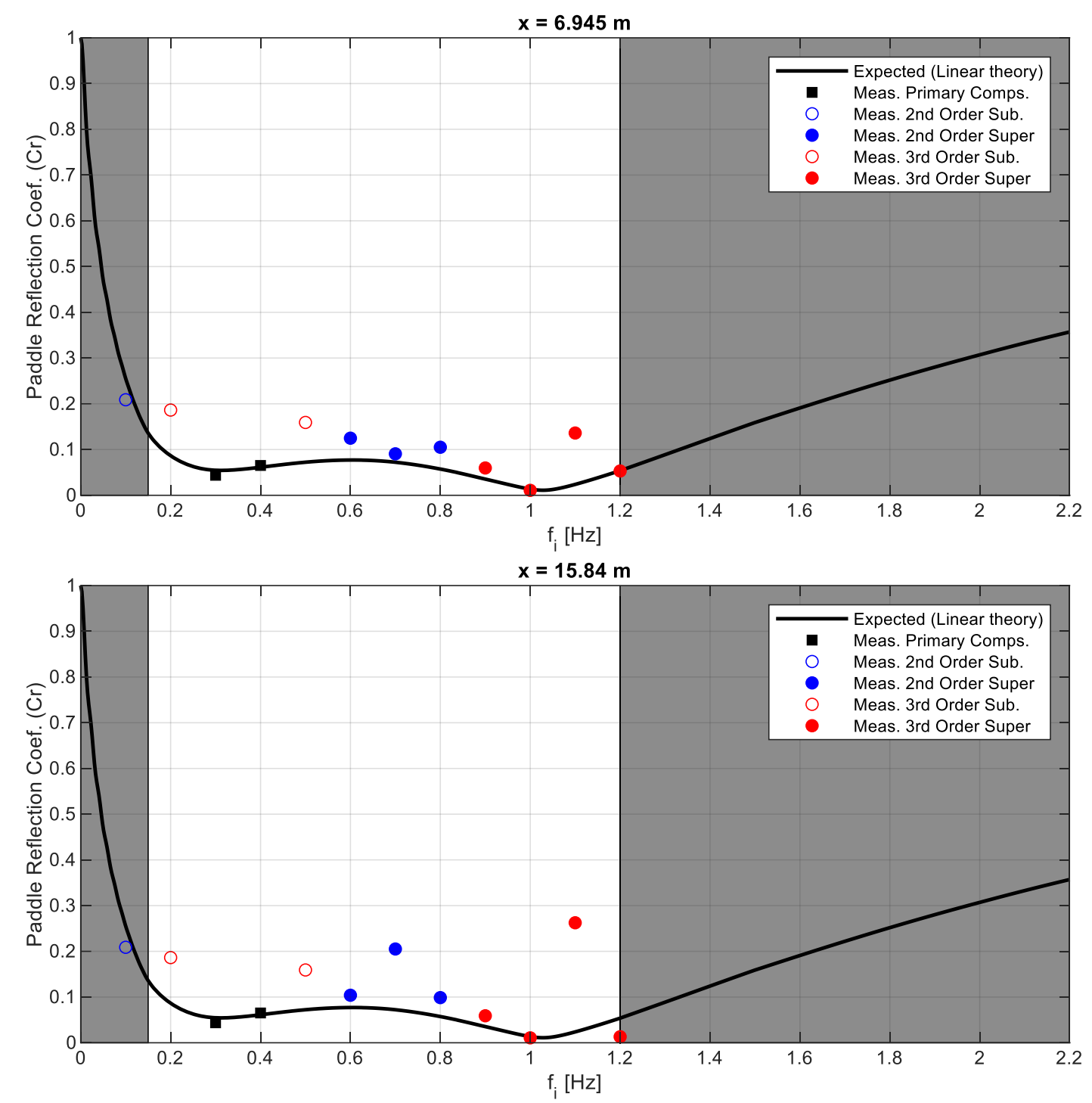

Figure 4. Comparison of reflection coefficients from test BIC01 with the linear absorption performance curve. 
This conclusion is further examined in Fig. 5 where the reflection coefficient is plotted for all twelve bichromatic test cases. Based on above findings significant scatter might be expected for components with very small incident and reflected amplitudes. Thus, only components with an incident amplitude larger than $5 \mathrm{~mm}$ are included in Fig. 5. The figure demonstrates that similar results are obtained based on total amplitudes at wavemaker 2 as in the wave gauge array, but with more scatter at the wavemaker especially for the higher frequencies where the spatial extrapolation is more difficult. The figure shows in general similar results as found for the regular waves in Fig. 1. Thus, it is fair to conclude that bound sub and superharmonics in bichromatic waves have similar absorption performance as a free wave at the same frequency.
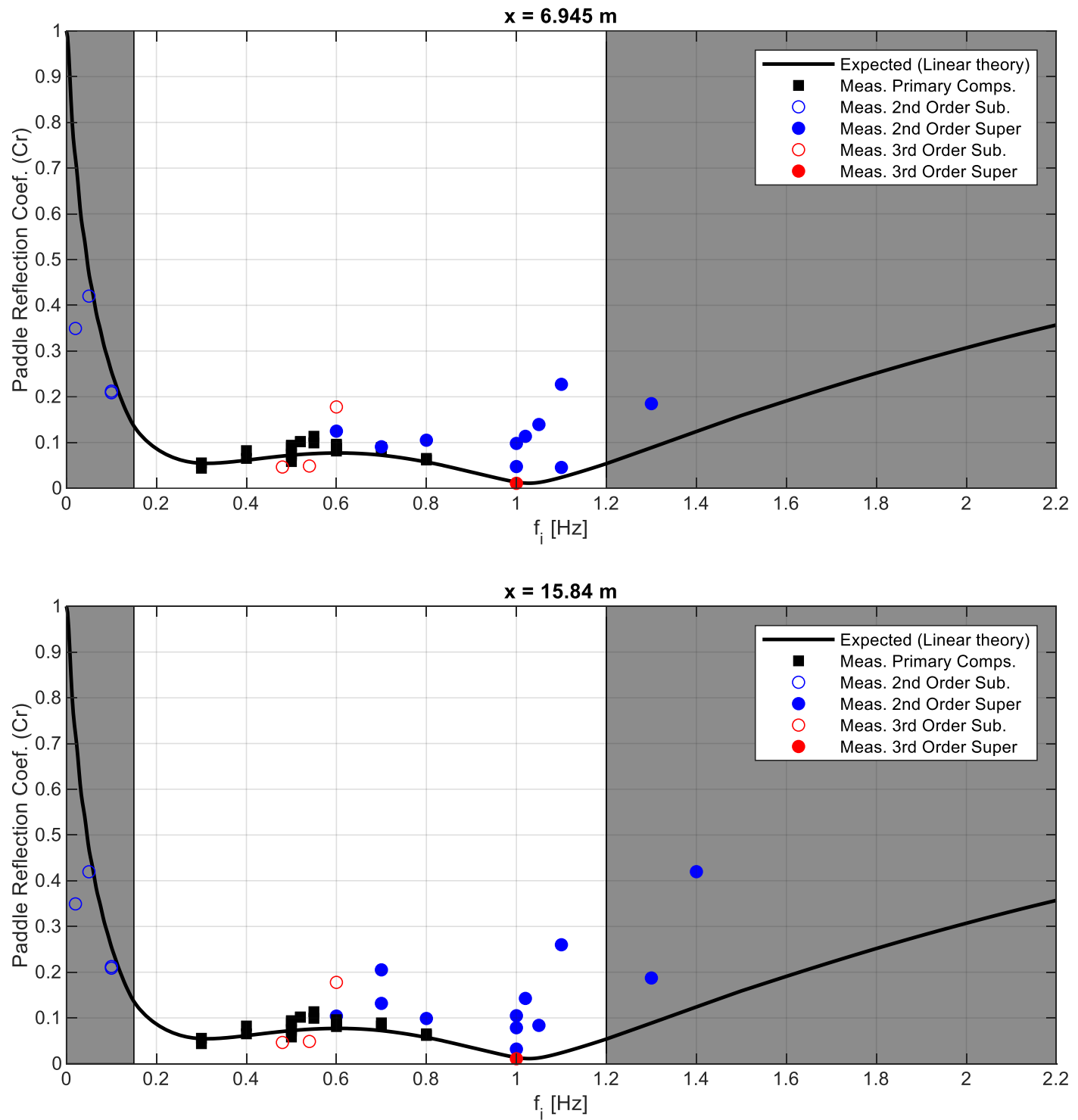

Figure 5. Similar as Fig. 4, but including all 12 tests with bichromatic waves. Components with an incident total amplitude less than $5 \mathrm{~mm}$ have been excluded. 


\section{RESULTS FOR NONLINEAR IRREGULAR WAVES}

For the irregular waves the reflection coefficient is calculated based on the estimated total amplitudes in the mean wave gauge position. This is because the spatial extrapolation to Wavemaker 2 might give larger errors for irregular waves as the bound harmonics are due to interaction of many frequencies. Based on the bichromatic wave tests this choice should provide reasonable estimates for the reflection at the paddle.

The results for the irregular waves appear in Fig. 6 for the three tests with a peak wave period $\left(T_{\mathrm{p}}\right)$ of $3 \mathrm{~s}$. SW03 is the most linear irregular sea state, and it appears that the estimated incident spectrum is in good agreement with the target JONSWAP spectrum. Additionally, the figure shows that the measured reflection coefficient as function of frequency is in agreement with the theoretical re-reflection coefficient of the paddle calculated based on linear wavemaker theory.
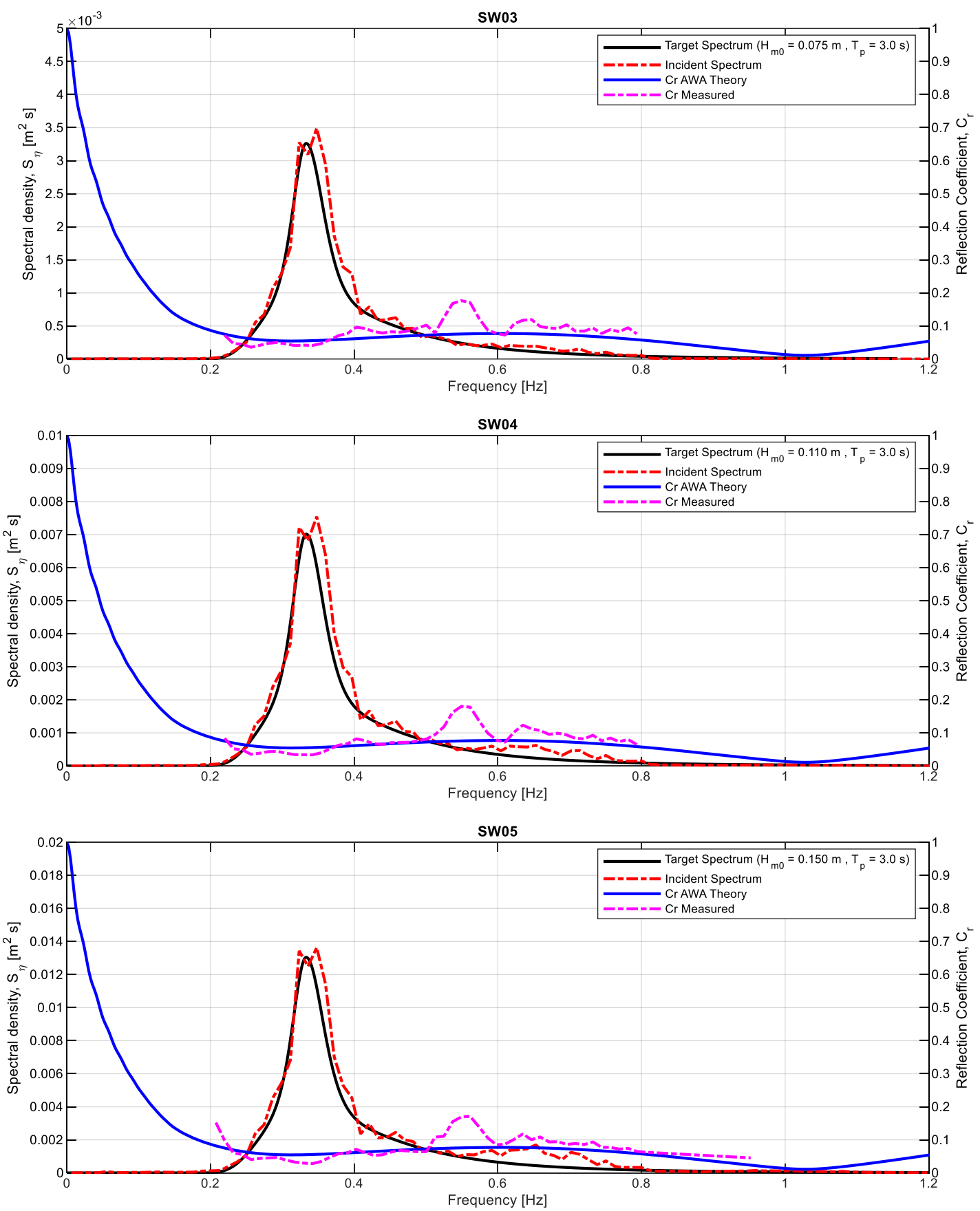

Figure 6. Spectral results for the three irregular tests (SW03-SW05) with peak period $T_{\mathrm{p}}=3 \mathrm{~s}$. 
The two other tests (SW04 and SW05) in Fig. 6 are with increasing wave height and thus increasing nonlinearities. For both these tests a significant $2^{\text {nd }}$ order superharmonic peak is observed, and for the most nonlinear test (SW05) the bound energy around $2 f_{\mathrm{p}}$ is higher than the primary energy (target spectrum). However, even in that case the determined reflection coefficient at the frequency band with high superharmonic energy does not increase compared to the more linear waves in test SW03. In all cases, the measured reflection coefficient agrees well with the theoretical absorption performance curve based on linear wavemaker theory.

Similar results are found in Fig. 7 for a peak wave period of $4 \mathrm{~s}$. This indicates that bound $2^{\text {nd }}$ order superharmonics in fully irregular waves behave similarly to the bound harmonics in the bichromatic and regular waves. Thus, the performance of the active absorption system on bound harmonics in irregular waves does not deviate significantly from a linear component at the same frequency.
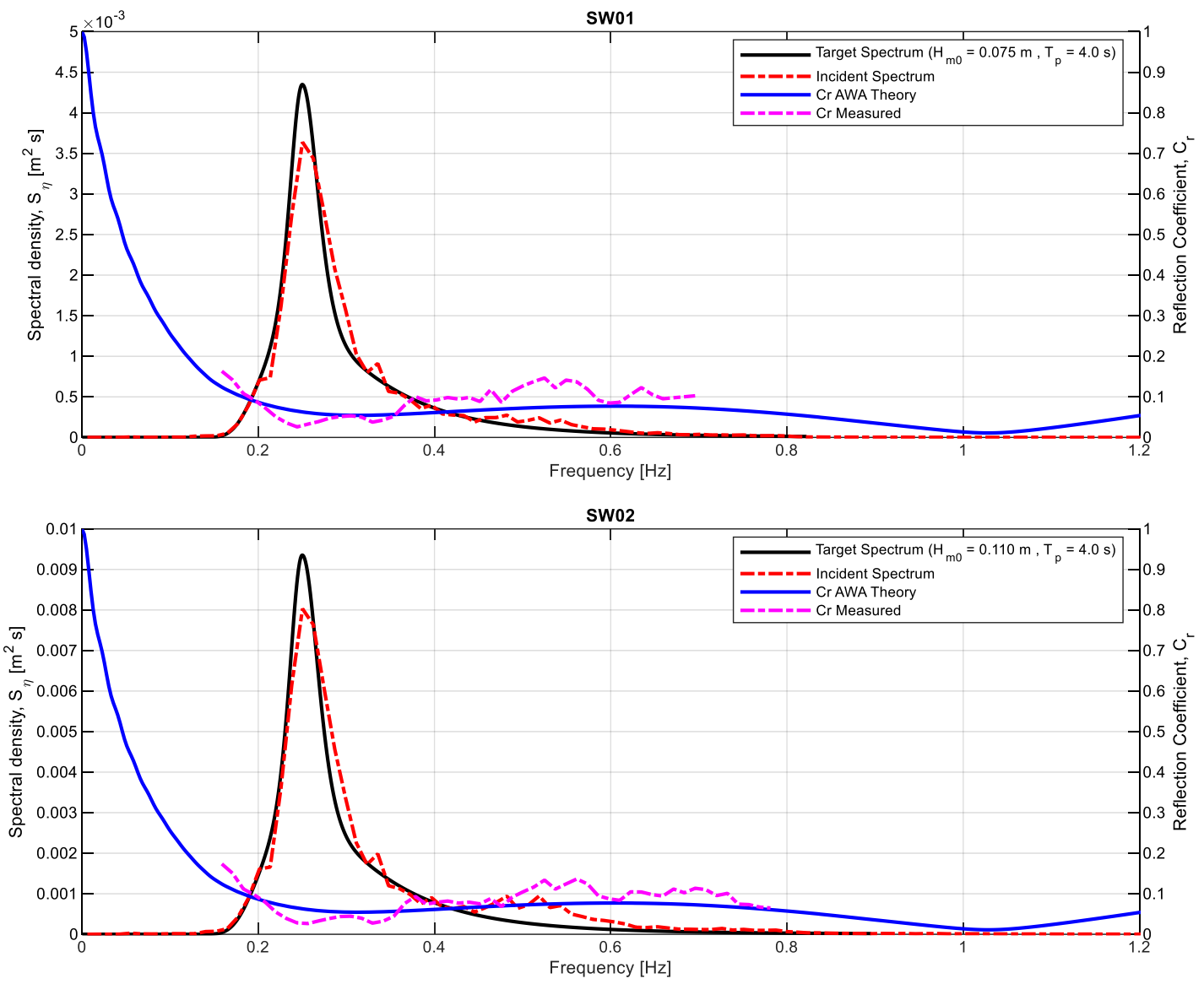

Figure 7. Spectral results for the two irregular tests (SW01, SW02) with peak period $T_{\mathrm{p}}=4 \mathrm{~s}$.

\section{CONCLUSIONS}

In hydraulic model tests, it is important to deal with reflections in a proper way. Otherwise, the incident wavefield might be contaminated with re-reflections from the basin boundaries. In order to minimize re-reflections at the wave generator, active absorption must be applied. An active absorption system will detect the reflected wave from the model and generate the opposite wave to cancel out the re-reflected one. Such systems have existed for more than 50 years and have been common practice for the major hydraulic laboratories for the last 25 years.

Even though active absorption has been common practice for many years the performance of such systems when applied to nonlinear waves has not been studied. The aim of the present study was thus to study the response of the Lykke Andersen et al. (2016) active absorption system for nonlinear waves. It has previously been demonstrated that their system is able to absorb bound superharmonics in regular waves with similar performance as a free component at the same frequency. In the present paper, this study is extended to bichromatic and irregular waves. 
For this purpose, new model tests were carried out in a wave flume with wavemakers in both ends. By determination of the re-reflected wave field from the second wavemaker the performance of the active absorption system was studied. The surface elevations were measured by an array of wave gauges and then separated into incident and reflected waves in order to calculate the reflection coefficient of the absorbing wavemaker. The very recently published methods to separate nonlinear bichromatic and irregular waves into incident and reflected components were used for this purpose.

The results show that the active absorption system is able to absorb the bound sub and super harmonics in bichromatic and irregular waves, with similar absorption performance as a free wave at the same frequency.

\section{REFERENCES}

Aalborg University (2018a). AwaSys webpage, www.hydrosoft.civil.aau.dk/awasys

Aalborg University (2018b). WaveLab webpage, www.hydrosoft.civil.aau.dk/wavelab

Biésel, F. and Suquet, F. (1951). Les Appareils generateurs de Houle en Laboratoire. La Houille Blanche, Vol. 6, nos. 2,4 et 5.

Chatry, G., Clement, A. H., Gouraud, T. (1998). Self-adaptive control of a piston wave absorber. Proceedings of the 8th Int. Offshore and Polar Eng. Conference, pp.127-133.

Clavero, M., Aguilera, L., Nieto, S., Longo, S. and Losada, M. A. (2013). Experimental study of the interaction atmosphere-ocean. XII Coast and Ports Spanish Conference. Cartagena (Spain). In Spanish.

Eldrup, M. R. and Lykke Andersen, T. (2018). Applicability of Nonlinear Wavemaker Theory. Proc. CoastLab2018 conference, Santander, Spain.

Eldrup, M. R. and Lykke Andersen, T. (2019). Estimation of Incident and Reflected Wave Trains in Highly Nonlinear Two-Dimensional Irregular Waves. Journal of Waterway, Port, Coastal, and Ocean Engineering, Vol. 145, Issue 1 (January 2019).

Frigaard, P. and Christensen, M. (1994). An Absorbing Wave-maker Based on Digital Filters. Proceedings of the 24th International Conference on Coastal Engineering, Kobe, Japan

Gilbert, G. (1978). Absorbing wave generators. Hydraulic Research Station, Note 20.

Hald, T. and Frigaard, P. (1996). Performance of Active Wave Absorption Systems: Comparison of Wave Gauge and Velocity Meter based Systems, Proc. 2nd International Conference on Coastal, Ports, and Marine Structures "ICOPMAS", Tehran, Iran.

Hald, T., Frigaard, P. (1997a). Performance of Active Wave Absorption Systems: Comparison of Wave Gauge and Velocity Meter based Systems. Proceedings of the 7th International Offshore and Polar Engineering Conference, ISOPE, Honolulu, Hawaii, May 1997.

Hald, T. and Frigaard, P. (1997b). Alternative Method for Active Absorption in Multidirectional Waves. Proceedings of the 27th IAHR Congress, San Francisco. IAHR Seminar: Multidirectional Waves and their Interaction with Structures.

Klopman, G., Reniers, A., Wouters, J. and de Haan, T., (1996). Active Multidirectional wave absorption. Abstract, 25th Int. Conf. on Coastal Eng., Orlando, Florida, USA.

Kostese, J. K. (1984). Measurements of beat and set-down beneath wave groups. Proc. Of the 19th Int. Conf. on Coastal Eng., Houston, Texas, USA, pp. 724-740.

Lykke Andersen, T. and Frigaard, P. (2014). Wave Generation in Physical Models: Technical documentation for AwaSys 6. DCE Lecture Notes, Vol. 34, Department of Civil Engineering, Aalborg University.

Lykke Andersen, T., Clavero, M., Frigaard, P., Losada, M. and Puyol, J. I. (2016). A new active absorption system and its performance to linear and non-linear waves. Coastal Engineering Vol.114, Pages 47-60.

Lykke Andersen, T., Eldrup, M. R. and Frigaard, P. (2017). Estimation of Incident and Reflected Components in Highly Nonlinear Regular Waves. Coastal Engineering, Vol. 119, pp 51-64.

Lykke Andersen, T., Eldrup, M.R., Clavero, M. (2018). Separation of Long-Crested Nonlinear Bichromatic Waves into Incident and Reflected Components. Proc. CoastLab2018 conference, Santander, Spain.

Madsen, P. A. and Fuhrman, D. R. (2006). Third-order theory for bicromatic bi-directional water waves. J. Fluid Mech., Vol. 557, pp. 369-397.

Madsen, P. A. and Fuhrman, D. R. (2012). Third-order theory for multi-directional irregular waves. J. Fluid Mech., Vol. 698, pp. 304-334. 
Maisondieu, C. and Clement, A. (1993). A realizable force feedback-feedforward control loop for a piston wave absorber. Proceedings of the 8th Int. Workshop on Water Waves and Floating bodies, St Johns Newfoundland, Canada, pp.79-82.

Milgram, J. H. (1965). Compliant Water Wave Absorbers. Massachusetts Institute of Technology, Department of Naval Architecture and Marine Engineering Research Report no. 65-13, Cambridge, Massachussetts.

Milgram, J.H. (1970), Active Water-wave Absorbers. J. Fluid. Mech., Vol. 43(4), pp 845-859.

Schäffer, H.A., Stolborg, T., Hyllested, P. (1994). Simultaneous Generation and Active Absorption of Waves in Flumes. Proceedings of Int. Symp.: Waves - Physical and Numerical Modelling, Vol. 1, Vancouver.

Schäffer, H.A. (2001). Active Wave Absorption in Flumes and 3D Basins. Proc. 4th Int. Symp. on Ocean Wave Measurement and Analysis, San Francisco, CA, USA, pp. 1200-1208.

Schäffer, H. A. and Jakobsen, K. P. (2003). Non-linear wave generation and active absorption in wave flumes. Proc. Long Waves Symposium, Thessaloniki, Greece, pp. 69-77.

Schäffer, H. A. and Steenberg, C. M. (2003). Second-order wavemaker theory for multidirectional waves. Ocean Engineering 30, pp 1203-1231.

Spinneken, J. and Swan, C. (2009). Wave Generation and Absorption Using Force-Controlled Wave Machines. Proc. 19th Int. Offshore and Polar Eng. Conf. (ISOPE), Osaka, Japan.

Zhang, H., Schäffer, H.A., Jakobsen, K.P. (2007). Deterministic combination of numerical and physical coastal wave models. Coastal Engineering 54, pp 171-186. 